\title{
The Importance of Chain-Length Dependent Kinetics in Free-Radical Polymerization: A Preliminary Guide
}

\author{
Johan P.A. Heuts, ${ }^{1}$ Gregory T. Russell, ${ }^{2}$ Gregory B. Smith, ${ }^{2}$ Alex M. van \\ Herk $^{1}$ \\ ${ }^{1}$ Laboratory for Polymer Chemistry, Department of Chemical Engineering \\ and Chemistry, Eindhoven University of Technology, PO Box 513, $5600 \mathrm{MB}$ \\ Eindhoven, The Netherlands; E-mail: j.p.a.heuts@tue.nl \\ ${ }^{2}$ Department of Chemistry, University of Canterbury, Private Bag 4800, \\ Christchurch, New Zealand
}

Summary: The effect of chain-length dependent propagation at short chain lengths on the observed kinetics in low-conversion free-radical polymerization (frp) is investigated. It is shown that although the values of individual propagation rate coefficients quickly converge to the high chain length value (at chain lengths, $i$, of about 10), its effect on the average propagation rate coefficients, $\left\langle k_{\mathrm{p}}\right\rangle$, in conventional frp may be noticeable in systems with an average degree of polymerization $\left(D P_{n}\right)$ of up to 100 . Furthermore it is shown that, unless the system is significantly retarded, the chain-length dependence of the average termination rate coefficient, $\left\langle k_{\mathrm{t}}\right\rangle$, is not affected by the presence of chain-length dependent propagation and that there exists a simple (fairly general) scaling law between $\left\langle k_{\mathrm{t}}\right\rangle$ and $D P_{\mathrm{n}}$. This latter scaling law is a good reflection of the dependence of the termination rate coefficient between two $i$ meric radicals, $k_{\mathrm{t}}^{i, i}$, on $i$. Although simple expressions seem to exist to describe the dependence of $\left\langle k_{\mathrm{p}}\right\rangle$ on $D P_{\mathrm{n}}$, the limited data available to date does not allow the generalization of these expressions.

Keywords: free-radical polymerization; kinetics; chain-length dependent propagation; chain-length dependent termination

\section{Introduction}

The main process and product parameters to be controlled in free-radical polymerization are the rate of polymerization $\left(R_{\mathrm{p}}\right)$ and the molecular weight distribution of the formed polymer. In the latter case, one often tries to control the number average degree of polymerization $\left(D P_{\mathrm{n}}\right)$ and the polydispersity index $(P D I)$. Although an increasing number of researchers are starting to use (complicated) computer modelling packages, most people would still use the steady-state rate equation (Eq. 1) for predicting changes in rate and the Mayo equation (Eq. 2) for predicting changes in the average degree of polymerization when changing reaction conditions. 
The steady-state rate equation for a free-radical polymerization of a monomer $\mathrm{M}$ initiated by a thermal initiator I, with decomposition rate coefficient $k_{\mathrm{d}}$ and initiator efficiency $f$ (defined as the fraction of primary radicals not undergoing cage reactions), is given by Eq. 1 , where $\left\langle k_{\mathrm{t}}\right\rangle$ is the chain-length averaged termination rate coefficient and $\left\langle k_{\mathrm{p}}\right\rangle$ is the chainlength averaged propagation rate coefficient for the given system. The use of a systemdependent $\left\langle k_{\mathrm{t}}\right\rangle$ instead of an (incorrect) single chain-length independent value of $k_{\mathrm{t}}$ in this equation seems to be generally accepted now, ${ }^{[1,2]}$ but as we have shown previously and will elaborate upon in this paper, in certain cases the use of $\left\langle k_{\mathrm{p}}\right\rangle$ instead of the long-chain $k_{\mathrm{p}}$ value is also required. ${ }^{[3-5]}$

$$
R_{\mathrm{p}}=\left\langle k_{\mathrm{p}}\right\rangle \sqrt{\frac{f k_{\mathrm{d}}[\mathrm{I}]}{\left\langle k_{\mathrm{t}}\right\rangle}}[\mathrm{M}]
$$

Similarly, the familiar Mayo equation, given by Eq. 2, should contain $\left\langle k_{\mathrm{p}}\right\rangle$ and $\left\langle k_{\mathrm{t}}\right\rangle$ instead of their chain-length independent equivalents.

$$
\frac{1}{D P_{\mathrm{n}}}=(1+\lambda) \frac{\left\langle k_{\mathrm{t}}\right\rangle[\mathrm{R}]}{\left\langle k_{\mathrm{p}}\right\rangle[\mathrm{M}]}+\sum_{\mathrm{X}} \frac{k_{\mathrm{tr}, \mathrm{X}}[\mathrm{X}]}{\left\langle k_{\mathrm{p}}\right\rangle[\mathrm{M}]}
$$

In this equation, $\lambda$ is the fraction of chains terminated by disproportionation, $[R]$ is the overall radical concentration and $k_{\mathrm{tr}, \mathrm{X}}$ is the rate coefficient for chain transfer to any chain transfer agent $X$ (including monomer). Note that a chain-length independent chain transfer rate coefficient has been used, which is unlikely to be the case for similar reasons as to why the propagation rate coefficient is chain-length dependent. ${ }^{[6]}$ However, in order to not unnecessarily overcomplicate the discussion and to focus on the effect of chainlength dependent propagation, we have assumed $k_{\mathrm{tr}, \mathrm{X}}$ independent of chain length in the current study.

Both equations are, in principle, simple to use and clearly show how the rate and molecular weight change with changing reaction conditions (i.e., reactant/additive concentrations and rate coefficients). The only complicating factor in using these expressions is the fact that adequate values for $\left\langle k_{\mathrm{t}}\right\rangle$ (and in some cases also for $\left\langle k_{\mathrm{p}}\right\rangle$ ) must be used and these values are not always readily available from standard reference sources such as the Polymer Handbook. ${ }^{[1]}$ In the case of $\left\langle k_{\mathrm{t}}\right\rangle$ this is caused by the fact that the reaction is diffusion-controlled and hence the rate coefficient for termination is chainlength dependent; therefore a chain-length averaged value, given by Eq. 3, should be used. 


$$
\left\langle k_{\mathrm{t}}\right\rangle=\frac{\sum_{i=1}^{\infty} \sum_{j=1}^{\infty} k_{\mathrm{t}}^{i, j}\left[\mathrm{R}_{i}\right]\left[\mathrm{R}_{j}\right]}{[\mathrm{R}]^{2}}
$$

In this expression, $k_{\mathrm{t}}^{\mathrm{i}, j}$ is the rate coefficient for the termination reaction between an $i$ meric radical $\mathrm{R}_{i}$ and a $j$-meric radical $\mathrm{R}_{j}$. It is important to note that in this work $\mathrm{R}_{1}$ refers to a truly monomeric radical, whether it has been derived from initiator, chain transfer agent or chain transfer to monomer (so it does not refer to the radical after the first addition to monomer - this radical would be denoted as $R_{2}$ here). Hence, to really determine a value for $\left\langle k_{t}\right\rangle$ one would need to know the individual values for the $k_{t}^{i, j}$ and the propagating radical distribution. It is therefore clear that a "termination rate coefficient" measured for a given monomer may not be applicable to the same monomer, polymerized under different reaction conditions. ${ }^{[1]}$ To make things even more complicated, $\left\langle k_{t}\right\rangle$ also depends on conversion, as the diffusion of the chains depends highly on the viscosity of the reaction medium. ${ }^{[1]}$ In order to simplify our discussion, we limit ourselves here to low-conversion polymerization, so as to eliminate this conversion/viscosity effect.

The chain-length dependence of the propagation rate coefficient is of a more "chemical" nature in that it is caused by differences in the activation energy and the frequency factor of the actual, intrinsic, rate coefficients of the addition reaction for different size radicals. ${ }^{[5]}$ The chain-length averaged propagation rate coefficient is defined by Eq. 4 ,

$$
\left\langle k_{\mathrm{p}}\right\rangle=\frac{\sum_{i=1}^{\infty} k_{\mathrm{p}}^{i}\left[\mathrm{R}_{i}\right]}{[\mathrm{R}]}
$$

where $k_{\mathrm{p}}{ }^{i}$ is defined as the rate coefficient for the addition of an $i$-meric radical to monomer. The chain-length dependence of $k_{\mathrm{p}}$ is relatively small and only noticeable for systems in which a relatively low $D P_{\mathrm{n}}$ is produced (see below) ${ }^{[5]}$ Hence, in contrast to reported values of $k_{\mathrm{t}}$, which are only applicable to very specific situations, carefully obtained values for $k_{\mathrm{p}}$ in general do represent a "true" physical, generally applicable, rate coefficient (be it for long-chain propagation).

So, where does this leave the experimental polymer chemist? Is detailed knowledge really required about $k_{\mathrm{p}}{ }^{i}, k_{\mathrm{t}}^{i, j}$ and the distribution of $\mathrm{R}_{i}$ ? Those familiar with the literature regarding chain-length dependent termination (and now also chain-length dependent propagation) have probably encountered unfriendly looking mathematical equations and 
some may have even decided to put the paper aside labelling it as only relevant to theoreticians. To some extent these readers might have been right in their thinking, were it not that chain-length dependence often causes deviations from what is expected from classical theory and ignoring it in certain instances can cause incorrect conclusions to be drawn. Hence, for those workers only interested in rough estimates for the chain-length dependence of $\left\langle k_{\mathrm{p}}\right\rangle$ and $\left\langle k_{\mathrm{t}}\right\rangle$ to be used in Eqs. 1 and 2, it would be very useful to have approximate scaling laws such as Eqs. 5 and 6.

$$
\begin{aligned}
& \left\langle k_{\mathrm{t}}\right\rangle \approx G \cdot D P_{\mathrm{n}}^{-e} \\
& \left\langle k_{\mathrm{p}}\right\rangle \approx Q \cdot D P_{\mathrm{n}}^{-a}
\end{aligned}
$$

Here, $G$ and $Q$ are constant pre-exponential factors and $e$ and $a$ scaling exponents for $\left\langle k_{\mathrm{t}}\right\rangle$ and $\left\langle k_{\mathrm{p}}\right\rangle$, respectively.

In what follows we will investigate whether such scaling laws exist and how important chain length dependent propagation is in free-radical polymerization.

\section{Chain-Length Dependent Termination and Propagation Rate Coefficients}

It has been known for many decades that the termination process is diffusion-controlled and therefore the rate coefficient for termination depends on the length of the reacting radical. $^{[1]}$ Furthermore, it has been known that the rate-determining processes for the termination of small and long radicals are center-of-mass and segmental diffusion, respectively. These processes scale with the chain length as $i^{-e}$, where $e \approx 0.5$ and 0.16 for the former and latter processes respectively. It is also known that two monomeric radicals undergo a termination reaction with a rate coefficient of about $10^{9} \mathrm{dm}^{3} \mathrm{~mol}^{-1} \mathrm{~s}^{-1}$. Although these facts have been known for quite some time, we recently presented for the first time a

simple composite termination model that encompasses all these experimental facts. ${ }^{[7]}$ In this model, which is schematically shown in Figure 1, the termination rate coefficient between two $i$-meric radicals is given by Eq. 7, where we assume a critical chain length $i_{\text {crit }}$ of about 100 units at which the rate determining process from center-of-mass diffusion $\left(i \leq i_{\text {crit }}\right)$ changes to segmental diffusion $\left(i>i_{\text {crit }}\right)$. Cross-termination is then described by $k_{\mathrm{t}}^{i, j}=\left(k_{\mathrm{t}}^{i, i} \times k_{\mathrm{t}}^{j, j}\right)^{1 / 2}$. 


$$
k_{\mathrm{t}}^{i, i}= \begin{cases}k_{\mathrm{t}}^{1,1} \times i^{-e_{\mathrm{S}}} & \text { for } i \leq i_{\text {crit }} \\ k_{\mathrm{t}}^{1,1} \times\left(i_{\text {crit }}\right)^{-\left(e_{\mathrm{S}}-e_{\mathrm{L}}\right)} \times i^{-e_{\mathrm{L}}} & \text { for } i>i_{\text {crit }}\end{cases}
$$

The values for the parameters in Eq. 7 that we used in our modeling for MMA at $60^{\circ} \mathrm{C}$ are $k_{\mathrm{t}}^{1,1}=1 \times 10^{9} \mathrm{dm}^{3} \mathrm{~mol}^{-1} \mathrm{~s}^{-1}, e_{\mathrm{S}}=0.50, e_{\mathrm{L}}=0.16$ and $i_{\text {crit }}=100$; we will use these parameters as our defaults in all the kinetic modelling for this paper. The applicability of this model was confirmed experimentally for several different monomer systems by Buback and coworkers with parameter-values very close to those proposed by us. ${ }^{[8,9]}$

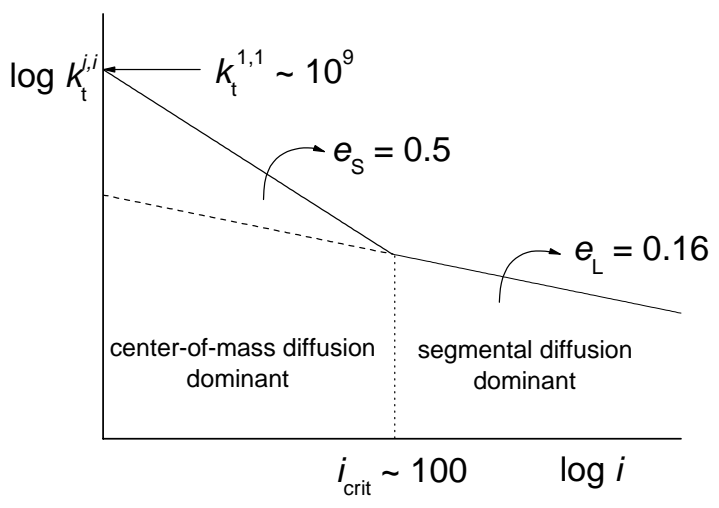

Figure 1. Chain-length dependence of $k_{\mathrm{t}}^{i, i}$ according to Eq. 7 indicating the regions where center-of-mass diffusion and segmental diffusion are the rate dominating processes.

Based on an analysis of kinetic data on small radical additions and the first few propagation steps in free-radical polymerization, backed up by theoretical investigations of the propagation rate coefficient, we proposed the empirical formula given by Eq. 8 for the description of the chain-length dependence of the propagation rate coefficient: ${ }^{[3-5]}$

$$
k_{\mathrm{p}}^{i}=k_{\mathrm{p}}\left[1+C_{1} \exp \left\{-\frac{\ln 2}{i_{1 / 2}}(i-1)\right\}\right]
$$

In this equation, $k_{\mathrm{p}}$ is the long-chain propagation rate coefficient, $C_{1}=\left(k_{\mathrm{p}}{ }^{1}-k_{\mathrm{p}}\right) / k_{\mathrm{p}}$ and $i_{1 / 2}$ is the chain length at which $k_{\mathrm{p}}{ }^{1}-k_{\mathrm{p}}$ halves in value (i.e., a sort of "half-life"). Available data thus far suggest $C_{1} \approx 10-50$ and $i_{1 / 2} \approx 0.5-1.5 ;^{[5]}$ for MMA polymerization we found values of $C_{1}=15.8$ and $i_{1 / 2}=1.12$. These latter values were obtained by fitting pulsed laser polymerization data obtained by Van Herk and co-workers ${ }^{[10]}$ and were found to describe well our (independently obtained) experimental steady state data (both rates and molecular weight distributions). ${ }^{[3,4]}$

In Figure 2, Eq. 8 is graphically displayed for $C_{1}=10$ and three different values for $i_{1 / 2}$, and it is clear from this figure that the chain length dependence of $k_{\mathrm{p}}{ }^{i}$ quickly converges to 
its long chain value: for the more realistic values of $i_{1 / 2}=0.5$ and 1.0, this happens before $i$ $=10$, and even for the unrealistically high value of $i_{1 / 2}=5$ this happens before $i=50$. This behaviour is not significantly affected by the value of $C_{1}$. Although this effect becomes insignificant quickly for the elemental rate coefficients, we will see in a following section that its macroscopic effect may be noticeable in polymerizations with average degrees of polymerization of up to 100 .

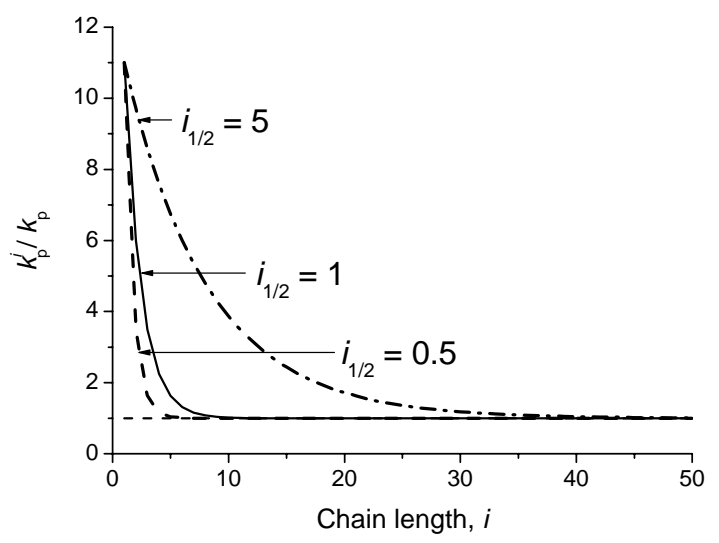

Figure 2. Chain-length dependence of $k_{\mathrm{p}}$ according to Eq. 8 , with $C_{1}=10$ and $i_{1 / 2}=0.5,1$ and 5.

Finally, two important notes need to be made here regarding chain length dependent propagation (CLDP): (i) the equation given by Eq. 8 is purely an empirical (but physically realistic!) formula that describes the currently available experimental and theoretical data well, and (ii) there is some contention as to whether there may be an additional process happening that causes an additional chain length dependence up to much higher chain lengths ${ }^{[10,11]}$ - in this work we limit ourselves to CLDP at short chain lengths.

\section{Kinetic Modelling Procedure}

In order to determine the values of $\left\langle k_{\mathrm{t}}\right\rangle$ and $\left\langle k_{\mathrm{p}}\right\rangle$ for varying reaction conditions, it can be seen from Eqs. 3 and 4 that we need to know the individual rate coefficients $k_{\mathrm{t}}{ }^{i, j}$ and $k_{\mathrm{p}}{ }^{i}$ and the radical distribution (i.e., $\left[\mathrm{R}_{i}\right]$ for all $i$ ). The individual rate coefficients are known from Eqs. 7 and 8, and the radical distribution can be determined using an iterative procedure for solving Eq. 9, which is easily derived after making the steady-state assumption for all radical concentrations. ${ }^{[7]}$

$$
\left[\mathrm{R}_{i}\right]=\frac{R_{\mathrm{init}}+f_{\mathrm{trX}}[\mathrm{R}]}{f_{\mathrm{p}}^{i}} \prod_{j=1}^{i}\left(\frac{f_{\mathrm{p}}^{j}}{f_{\mathrm{p}}^{j}+f_{\mathrm{trX}}+f_{\mathrm{t}}^{j}}\right) \quad \text { for } i=1, \infty
$$

In this equation, $R_{\text {init }}$ is the initiation rate $\left(=2 f k_{\mathrm{d}}[\mathrm{I}]\right.$ for a thermal initiator), $[\mathrm{R}]$ is the 
overall radical concentration, $f_{\mathrm{trx}}$ is the transfer frequency of an $i$-meric propagating radical $\left(=k_{\mathrm{trX}}[\mathrm{X}]\right), f_{\mathrm{p}}{ }^{i}$ is its propagation frequency $\left(=k_{\mathrm{p}}{ }^{i}[\mathrm{M}]\right)$ and $f_{\mathrm{t}}^{i}$ its termination frequency $\left(=\left(2 k_{\mathrm{t}}^{i, i} R_{\text {init }}\right)^{1 / 2}\right.$ for $\left.k_{\mathrm{t}}^{i, j}=\left(k_{\mathrm{t}}^{i, i} \times k_{\mathrm{t}}^{j, j}\right)^{1 / 2}\right)$. All these parameters are known, except the overall radical concentration $[R]$, which is at the same time an input of the calculation process and its result $\left([R]=\Sigma\left[R_{i}\right]\right)$. Hence, an iterative procedure is required to solve the radical balances, in which first a guess needs to be made for [R] (a reasonable starting point being a guess based on "classical" kinetics) after which Eq. 9 is solved up to sufficiently high $i$. Once convergence has been reached for $[R],\left\langle k_{t}\right\rangle$ and $\left\langle k_{\mathrm{p}}\right\rangle$ can be calculated using Eqs. 3 and 4. To get an exact value for the corresponding $D P_{\mathrm{n}}$ in the system, one would need to evaluate the entire molecular weight distribution starting from the radical distribution. Alternatively, one could use the Mayo equation (Eq. 2) and for short chains add 1 unit to the $D P_{n}$ to correct for the long-chain-approximation; although this is clearly an approximation, it is sufficiently accurate for the present purposes. This whole procedure, which we carried out using an EXCEL spreadsheet up to $i=65519$ (i.e., the maximum number of rows that we could use), is schematically shown in Figure $3 .^{[5]}$ In order to effect changes in $D P_{\mathrm{n}}$, we varied $f_{\text {trX }}$ and/or $R_{\text {init. }}$.

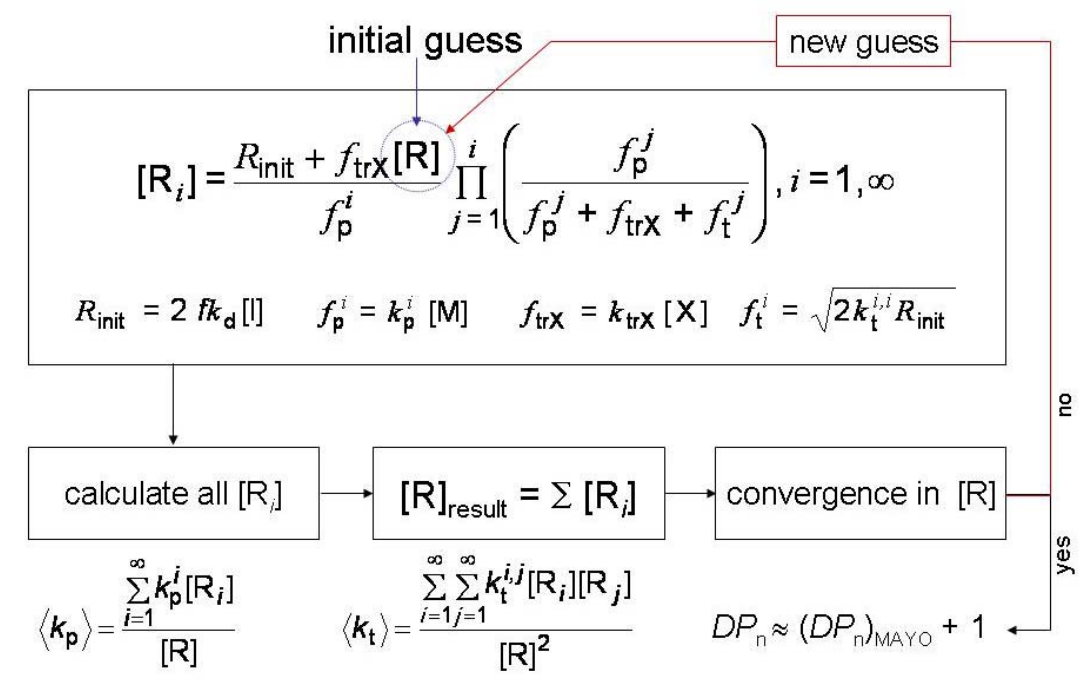

Figure 3. Schematic diagram containing the steps taken to determine $\left\langle k_{\mathrm{p}}\right\rangle$ and $\left\langle k_{\mathrm{t}}\right\rangle$ for systems with a varying $D P_{\mathrm{n}}$.

\section{The Effect of CLDP on the Observed Kinetics}

Firstly we will consider the effect of CLDP on the observed termination rate coefficient $\left\langle k_{\mathrm{t}}\right\rangle$. In Figure 4, the variation of $\left\langle k_{\mathrm{t}}\right\rangle$ with $D P_{\mathrm{n}}$ is shown for both chain length independent (CLIP) and dependent propagation. Two things are immediately clear from this figure. 
Firstly that the $\left\langle k_{\mathrm{t}}\right\rangle-D P_{\mathrm{n}}$ relationship reflects that of $k_{\mathrm{t}}^{i, i}-i$, and secondly that the effect of chain length dependent propagation on this relationship is very small. So, we can conclude that a simple scaling law exists between $\left\langle k_{\mathrm{t}}\right\rangle$ and $D P_{\mathrm{n}}$. Such a scaling law, holding for Eq. 7 with the given parameter values, is shown in Figure 4.

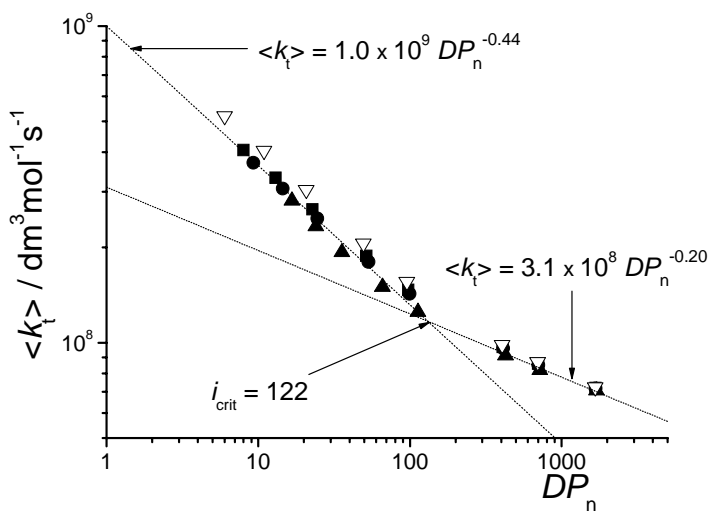

Figure 4. The chain-length dependence of the average termination rate coefficient assuming a constant $k_{\mathrm{p}}(\nabla)$ and chain length dependent $k_{\mathrm{p}}$ with $C_{1}=10$ and $i_{1 / 2}=0.5(\boldsymbol{\square})$, $1.0(\mathbf{O})$ and $5(\mathbf{\Delta})$.

In Figure 5, the relationship between the observed propagation rate coefficient $\left\langle k_{\mathrm{p}}\right\rangle$ and $D P_{\mathrm{n}}$ is shown. The first thing that draws attention is the fact that the effect of CLDP on $\left\langle k_{\mathrm{p}}\right\rangle$ is noticeable up to much higher values of $D P_{\mathrm{n}}$ than the value of the chain length $i$ up to which CLDP is significant in the individual rate coefficients (see Figure 2). For example, for the experimentally most likely values of $i_{1 / 2}=0.5$ and $1.0, k_{\mathrm{p}}{ }^{i} \approx k_{\mathrm{p}}$ for $i \approx 10$, but $\left\langle k_{\mathrm{p}}\right\rangle \approx k_{\mathrm{p}}$ only for $D P_{\mathrm{n}} \approx 100$. Hence, especially when working in systems where $D P_{\mathrm{n}}<$ 100 , one should be aware that the observed propagation rate coefficient $\left\langle k_{\mathrm{p}}\right\rangle$ may not be the same as the long chain propagation rate coefficient $k_{\mathrm{p}}$ (normally determined by PLP).
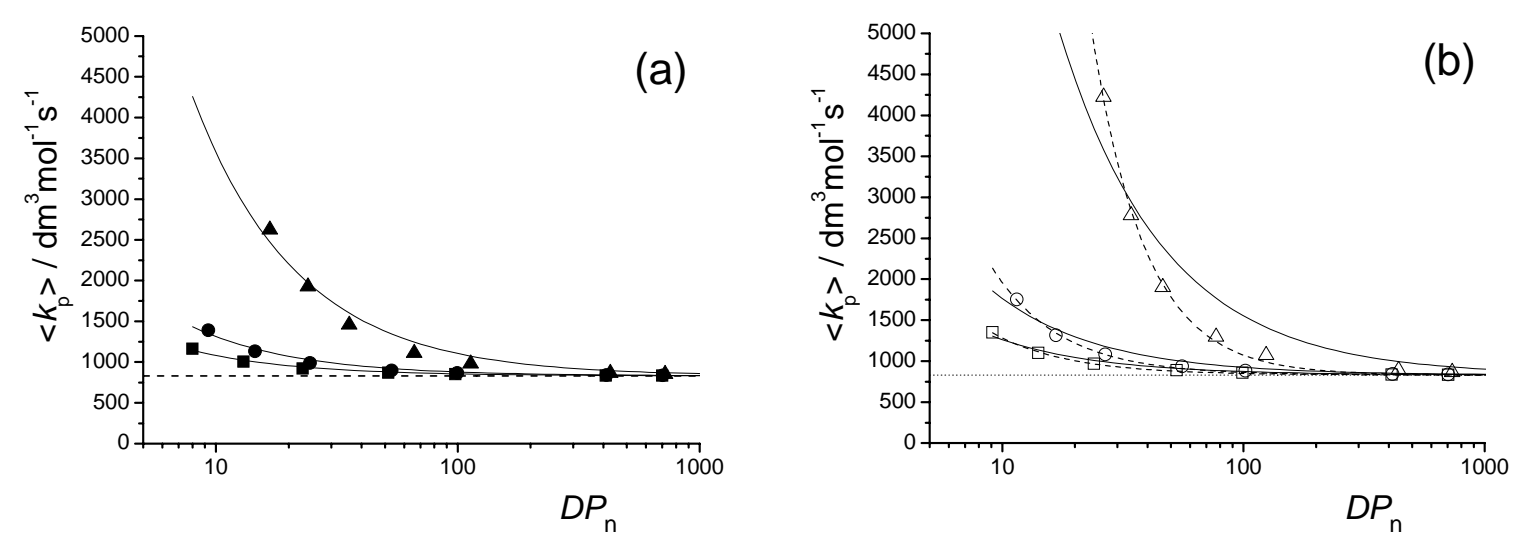

Figure 5. Dependence of $\left\langle k_{\mathrm{p}}\right\rangle$ on $D P_{\mathrm{n}}$, with (a) $C_{1}=10$ and $i_{1 / 2}=0.5(\mathbf{\square}), 1.0(\mathbf{O})$ and 5 (A); (b) with $C_{1}=50$ and $i_{1 / 2}=0.5(\square), 1.0(\bigcirc)$ and $5(\triangle)$. Full and dotted lines are the 
fits according to Eqs. 12 and 13 respectively.

In Figure 5 are also shown the first attempts to arrive at a simple scaling law for $\left\langle k_{\mathrm{p}}\right\rangle$ with $D P_{\mathrm{n}}$ similar to what was done earlier for $\left\langle k_{\mathrm{t}}\right\rangle$. Starting from a simple "two-state" propagation model used by Van Herk and co-workers (Eq. 10), ${ }^{[10]}$ we derived a linear relationship between $\left\langle k_{\mathrm{p}}\right\rangle$ and the amount of chain transfer agent in the system (Eq. 11). ${ }^{[4]}$

$$
\begin{aligned}
& k_{\mathrm{p}}^{i}= \begin{cases}\infty & \text { for } i \leq i_{\text {fast }} \\
k_{\mathrm{p}} & \text { for } i>i_{\text {fast }}\end{cases} \\
& \left\langle k_{\mathrm{p}}\right\rangle=k_{\text {trX }} \frac{[\mathrm{X}]}{[\mathrm{M}]} \times i_{\text {fast }}+k_{\mathrm{p}}
\end{aligned}
$$

The form of Eq. 11 suggests the possible existence of the following relationship between $\left\langle k_{\mathrm{p}}\right\rangle$ and $D P_{\mathrm{n}}$, where $Q^{\prime}$ is the only adjustable parameter.

$$
\left\langle k_{\mathrm{p}}\right\rangle=Q^{\prime} D P_{\mathrm{n}}^{-1}+k_{\mathrm{p}}
$$

The fits to the data with $C_{1}=10$ are shown in Figure $5 \mathrm{a}$ and the results appeared very promising, but in the case of $C_{1}=50$, the results were significantly worse as shown in Figure $5 b$.

Clearly, the simple propagation model (Eq. 10) on which Eq. 12 is based does not adequately describe the true CLDP behaviour and therefore we modified it to incorporate two fit parameters $Q$ and $a$ (Eq. 13). The corresponding data fits are also shown in Figure $5 \mathrm{~b}$ and it is immediately clear that Eq. 13 performs much better in describing the data than does Eq. 12. In Table 1, all fit parameters for Eqs. 12 and 13 to all combinations of $C_{1}=10,20$ and 50 and $i_{1 / 2}=0.5,1.0$ and 5.0 are listed.

$$
\left\langle k_{\mathrm{p}}\right\rangle=Q D P_{\mathrm{n}}^{-a}+k_{\mathrm{p}}
$$

Table 1. Summary of fit parameters for $\left\langle k_{\mathrm{p}}\right\rangle$ according to eqs 12 and 13.

\begin{tabular}{ccccc}
\hline$C_{1}$ & $i_{1 / 2}$ & $Q^{\prime} / \mathrm{dm}^{3} \mathrm{~mol}^{-1} \mathrm{~s}^{-1}$ & $Q / \mathrm{dm}^{3} \mathrm{~mol}^{-1} \mathrm{~s}^{-1}$ & $a$ \\
\hline 10 & 0.5 & $2.8 \times 10^{3}$ & $6.5 \times 10^{3}$ & 1.34 \\
10 & 1.0 & $5.7 \times 10^{3}$ & $2.1 \times 10^{4}$ & 1.49 \\
10 & 5.0 & $3.9 \times 10^{4}$ & $6.1 \times 10^{5}$ & 1.79 \\
20 & 0.5 & $3.4 \times 10^{3}$ & $8.4 \times 10^{3}$ & 1.37 \\
20 & 1.0 & $7.2 \times 10^{3}$ & $2.8 \times 10^{4}$ & 1.51 \\
\hline
\end{tabular}




\begin{tabular}{lllll}
\hline 20 & 5.0 & $5.3 \times 10^{4}$ & $1.1 \times 10^{6}$ & 1.87 \\
50 & 0.5 & $4.3 \times 10^{3}$ & $1.1 \times 10^{4}$ & 1.37 \\
50 & 1.0 & $9.3 \times 10^{3}$ & $3.9 \times 10^{4}$ & 1.54 \\
50 & 5.0 & $7.2 \times 10^{4}$ & $2.1 \times 10^{6}$ & 1.97
\end{tabular}

From Table 1 it can be seen that for the same value of $i_{1 / 2}, Q^{\prime}$ and $Q$ increase with increasing $C_{1}$ (as expected) and that $a$ increases with increasing $i_{1 / 2}$ (with only a small dependence on $C_{1}$ ). It would be useful to have a simple relationship between these fit parameters and the more "fundamental" CLDP parameters $C_{1}$ and $i_{1 / 2}$, but thus far we have not been able to discover any obvious one. (NB. Although $C_{1}$ and $i_{1 / 2}$ are indeed more fundamental in that they describe the chain length dependence of $k_{\mathrm{p}}{ }^{i}$, one should remember that, at least at present, Eq. 8 is also an empirical relationship).

We conclude this section with a discussion on the effect of CLDP on the observed rate of polymerization. In Figure 6, the dependence of $\left\langle k_{\mathrm{p}}\right\rangle /\left\langle k_{\mathrm{t}}\right\rangle^{1 / 2}$ (note that $R_{\mathrm{p}} \propto\left\langle k_{\mathrm{p}}\right\rangle /\left\langle k_{\mathrm{t}}\right\rangle^{1 / 2}$ ) on $D P_{\mathrm{n}}$ is shown, where the dotted line indicates the situation of CLIP.

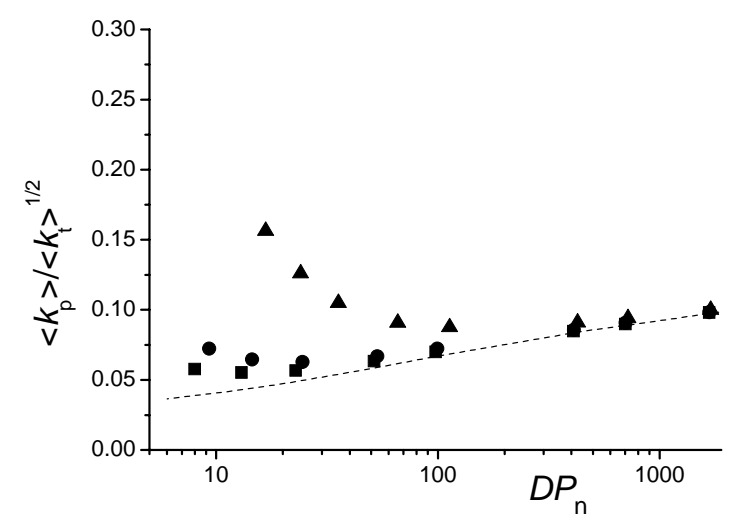

Figure 6. The effect of CLDP on the observed rate of polymerization (expressed here as $\left\langle k_{\mathrm{p}}\right\rangle /\left\langle k_{\mathrm{t}}\right\rangle^{1 / 2}$ ) at a given average degree of polymerization in the system. Data are shown for the cases of $C_{1}=10$ and $i_{1 / 2}=0.5(\boldsymbol{\square}), 1.0(\mathbf{O})$ and $5(\mathbf{\Delta})$.

As expected for CLIP, the ratio $\left\langle k_{\mathrm{p}}\right\rangle /\left\langle k_{\mathrm{t}}\right\rangle^{1 / 2}$ (and hence the rate) decreases with decreasing $D P_{\mathrm{n}}:\left\langle k_{\mathrm{t}}\right\rangle$ increases with decreasing $D P_{\mathrm{n}}$, while $k_{\mathrm{p}}$ remains constant. For CLDP we see a positive deviation from the CLIP situation, because the effect of an increasing value of $\left\langle k_{\mathrm{t}}\right\rangle$ is compensated by an increasing value of $\left\langle k_{\mathrm{p}}\right\rangle$ with decreasing $D P_{\mathrm{n}}$. This effect becomes more pronounced with increasing values of $i_{1 / 2}$ and $C_{1}$. It should also be noted here that this behaviour was observed experimentally for the low-conversion bulk polymerization 
of methyl methacrylate at $60^{\circ} \mathrm{C}$ in the presence of dodecanethiol. ${ }^{[3]}$

The main message from Figure 6 is that we will see different rate behaviour with changing $D P_{\mathrm{n}}$ depending on the values of $C_{1}$ and $i_{1 / 2}$; systems with a very weak dependence of $\left\langle k_{\mathrm{p}}\right\rangle$ on $D P_{\mathrm{n}}$ will show a decrease in rate at low $D P_{\mathrm{n}}$, whereas a stronger dependence may lead to apparent classical (chain-length independent) kinetics or even increased rates. Hence, when predicting the rate at lower values of $D P_{\mathrm{n}}$ from rate data at higher $D P_{\mathrm{n}}$ we may significantly underestimate the rate if we only take into account the chain length dependence of $\left\langle k_{t}\right\rangle$. It is therefore important to have an idea about the chain length dependence of either $k_{\mathrm{p}}$ or $\left\langle k_{\mathrm{p}}\right\rangle$. However, as is clear from Figures 4-6, any possible effects from CLDP probably only manifest themselves for $D P_{\mathrm{n}}<100$ and are probably safely ignored at higher $D P_{\mathrm{n}}$.

\section{The Effect of $\boldsymbol{k}_{\mathrm{p}}{ }^{1}$ on the Observed Kinetics}

Thus far, we have considered the chain length dependence of propagation assuming that $\mathrm{R}_{1}$ has the same, or a very similar, chemical nature as the polymeric propagating radical, i.e., it is a truly monomeric radical. Naturally, this need not always be the case. Initiatorderived radicals may react faster with a given monomer than the radical derived from this monomer, similar to propagating radicals that may prefer crosspropagation over homopropagation in copolymerization. The opposite can also be the case. One may have chosen a poor initiator and the primary radical reacts only slowly with monomer, e.g., cyanoisopropyl radical addition to vinyl acetate monomer. ${ }^{[12]}$ Additionally, chain transfer agent-derived radicals may reinitiate at different rates with different monomers, where slow additions can lead to retardation or inhibition as has recently been studied extensively in RAFT polymerization. ${ }^{[13]}$ It is therefore interesting to investigate the effect of different values of $k_{\mathrm{p}}{ }^{1}$ on the overall reaction kinetics; preliminary results of these studies have been published earlier and it should be noted that in this previous publication a small error was made in the calculation of $D P_{n} \cdot{ }^{[5]}$ Although this does not affect any qualitative conclusions of the earlier study, it changes the quantitative trends slightly. The results presented in this paper replace those presented earlier. ${ }^{[5]}$

We consider two different primary radicals $R_{A}$ and $R_{B}$, derived from initiator decomposition and chain transfer, respectively. The addition to monomer for these two 
radicals occurs with different rate coefficients as indicated in Scheme 1. For simplicity we assume that the resulting radicals after the first addition steps are indistinguishable and that the rate coefficient of the subsequent monomer addition is independent of the primary radical fragment. We realise that this assumption is unlikely to be completely correct as the existence of significant penultimate unit effects has been proven. ${ }^{[14]}$ However, it is unlikely that a possible penultimate unit effect will significantly alter any observed trends in CLDP and if so, it is expected that it would enhance the observed effect. Hence, while lacking any reliable quantitative information on the penultimate unit effect we assume Scheme 1 to be an adequate reflection of the kinetic situation.

$$
\left.\begin{array}{ll}
\mathrm{I} \stackrel{f k_{\mathrm{d}}}{\longrightarrow} 2 \mathrm{R}_{\mathrm{A}} & \mathrm{R}_{\mathrm{A}}+\mathrm{M} \stackrel{k_{\mathrm{p}}^{\mathrm{A}}}{\longrightarrow} \mathrm{R}_{2} \\
\mathrm{R}_{i}+\mathrm{X} \stackrel{k_{\mathrm{trX}}^{\mathrm{B}}}{\longrightarrow} \mathrm{P}_{i}+\mathrm{R}_{\mathrm{B}} & \mathrm{R}_{\mathrm{B}}+\mathrm{M} \stackrel{k_{\mathrm{p}}}{\longrightarrow} \mathrm{R}_{2}
\end{array}\right\} \mathrm{R}_{2}+\mathrm{M} \stackrel{k_{\mathrm{p}}^{2}}{\longrightarrow} \mathrm{R}_{3}
$$

\section{Scheme 1}

In the current study (using a modification of Eq. 9 to incorporate two different primary radicals as described previously), ${ }^{[5]} D P_{\mathrm{n}}$ was varied by varying the chain transfer frequency and we examined the effect of changing $k_{\mathrm{p}}{ }^{\mathrm{B}}\left(50 \times, 10 \times, 1 \times\right.$ and $\left.0.1 \times k_{\mathrm{p}}\right)$, while maintaining ${k_{\mathrm{p}}}^{\mathrm{A}}=(15.8+1) \times k_{\mathrm{p}}\left(\right.$ i.e., MMA at $\left.60^{\circ} \mathrm{C}\right)$; see Figure $7 \mathrm{a}$. In Figures $7 \mathrm{~b}-\mathrm{d}$, the results of these calculations are shown and it is immediately clear that only the lowest value of $k_{\mathrm{p}}{ }^{\mathrm{B}}$ gives results which are very different to those discussed in Figures 4-6.
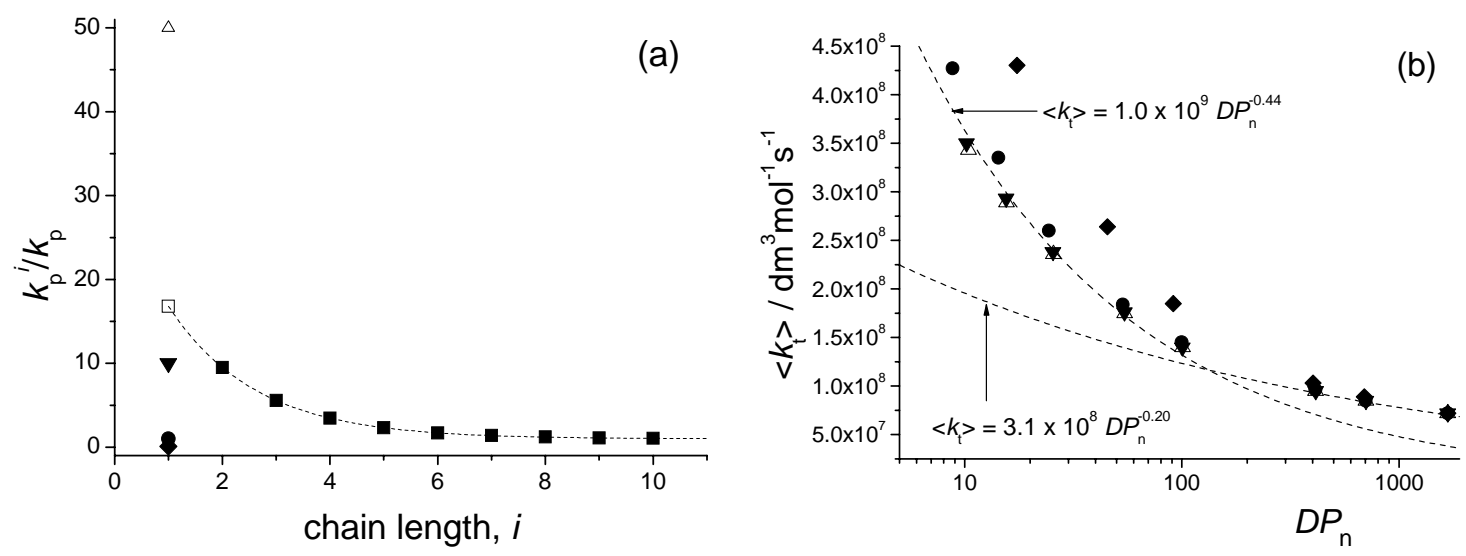

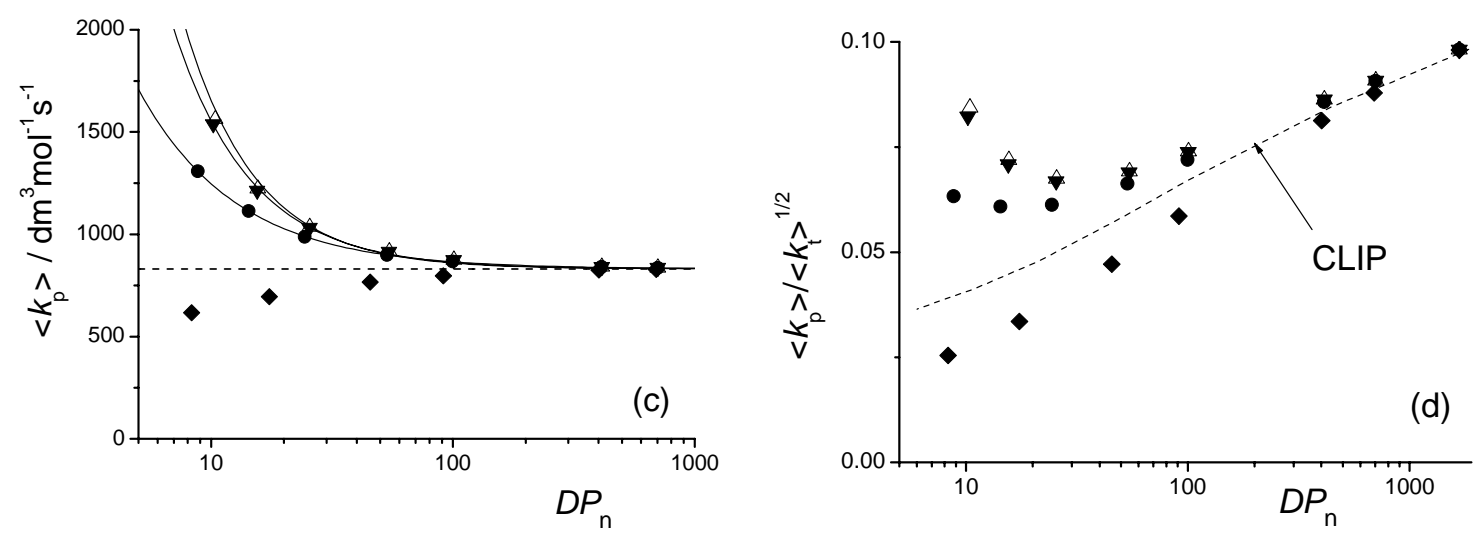

Figure 7. Effect of changing $k_{\mathrm{p}}{ }^{1}$ on the observed kinetics. Chain length dependence of (a) $k_{\mathrm{p}}{ }^{i}$, (b) $\left\langle k_{\mathrm{t}}\right\rangle$, (c) $\left\langle k_{\mathrm{p}}\right\rangle$ with full lines fits according to Eq. 13, (d) $\left\langle k_{\mathrm{p}}\right\rangle /\left\langle k_{\mathrm{t}}\right\rangle^{1 / 2}$. For all figures:

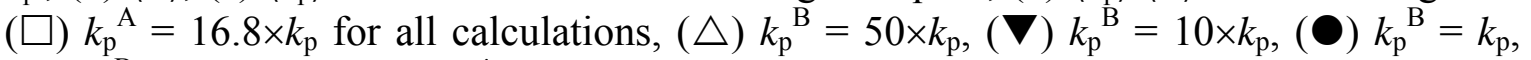
$(\bullet) k_{\mathrm{p}}^{\mathrm{B}}=0.1 \times k_{\mathrm{p}}$ and $(\square) k_{\mathrm{p}}^{i}$ according to Eq. (8) with $C_{1}=15.8$ and $i_{1 / 2}=1.12$ for all $i \geq$ 2, with $k_{\mathrm{p}}=831 \mathrm{dm}^{3} \mathrm{~mol}^{-1} \mathrm{~s}^{-1}$.

The calculated values of $\left\langle k_{\mathrm{t}}\right\rangle$ at low $D P_{\mathrm{n}}$ for $k_{\mathrm{p}}{ }^{\mathrm{B}}=0.1 \times k_{\mathrm{p}}$ are significantly higher than those for the other three cases, which are well described by the $\left\langle k_{t}\right\rangle$ equation derived from the data in Figure 4. This is presumably caused by an increase in primary radical termination, but more detailed simulations will be required to shed more light on this situation. In accordance with what we have seen earlier (i.e., in Figures 5-6 for $i_{1 / 2}=1$ ), the results in Figures $7 \mathrm{c}$ and $\mathrm{d}$ show that for all four values of $k_{\mathrm{p}}{ }^{\mathrm{B}}$ there is a significant effect on $\left\langle k_{\mathrm{p}}\right\rangle$ and the rate for $D P_{\mathrm{n}}<100$, with the results obtained for $k_{\mathrm{p}}{ }^{\mathrm{B}}=0.1 \times k_{\mathrm{p}}$ showing a very strong retardation. It is conceivable that this retardation effect is underestimated here, as a possible penultimate unit effect is likely to lower $k_{\mathrm{p}}{ }^{2}$ and hence further reduce $\left\langle k_{\mathrm{p}}\right\rangle$ and the rate at lower values of $D P_{\mathrm{n}}$. The data obtained for the other three cases were fitted by Eq. 13 with the resulting fit parameters listed in Table 2.

Table 2. Fit parameters according to Eq. (13) for the $\left\langle k_{\mathrm{p}}\right\rangle$ data in Figure 7c.

\begin{tabular}{ccc}
\hline$k_{\mathrm{p}}^{\mathrm{B}} / k_{\mathrm{p}}$ & $Q / \mathrm{dm}^{3} \mathrm{~mol}^{-1} \mathrm{~s}^{-1}$ & $a$ \\
\hline 0.1 & no fit possible & no fit possible \\
1.0 & $5.0 \times 10^{3}$ & 1.08 \\
10 & $1.7 \times 10^{4}$ & 1.34 \\
50 & $2.4 \times 10^{4}$ & 1.46
\end{tabular}

It can be seen from Figure 7c that Eq. 13 provides a reasonable description of the found 
$\left\langle k_{\mathrm{p}}\right\rangle$ data, with the situations in which $k_{\mathrm{p}}{ }^{\mathrm{B}}>k_{\mathrm{p}}$ having values for $Q$ and $a$ in the same range as those shown in Table 1 for $i_{1 / 2}=0.5-1$. Although it is too early to draw any general conclusions at this stage, the current results suggest that it is likely that in the future (with more explicit experimental data available) it may be possible to simply estimate the $\left\langle k_{\mathrm{p}}\right\rangle$ $D P_{\mathrm{n}}$ behaviour from a known value of $k_{\mathrm{p}}{ }^{1}$ and a generally assumed chain-length dependence of $k_{\mathrm{p}}{ }^{i}$.

In the light of the results discussed above, the rate data shown in Figure $7 \mathrm{~d}$ do not show any surprises. The case of $k_{\mathrm{p}}{ }^{\mathrm{B}}=0.1 \times k_{\mathrm{p}}$ shows a significant retardation at low $D P_{\mathrm{n}}$, whereas the other three cases show a faster rate as compared to the case of CLIP; in the cases where $k_{\mathrm{p}}{ }^{\mathrm{B}}>k_{\mathrm{p}}$ we observe a significant rate increase at low $D P_{\mathrm{n}}$.

\section{Conclusion}

In this paper we examined the effect of CLDP on kinetics in low-conversion free-radical polymerization. We have shown that although the chain length dependence of the individual $k_{\mathrm{p}}{ }^{i}$ does not extend beyond $i \approx 10$ for common systems, a significant macroscopic effect may be observed in systems with $D P_{n}$ up to $\sim 100$. This observation leads us to draw some preliminary conclusions regarding CLDP: (a) it should probably not be ignored in living radical polymerizations with low $D P_{\mathrm{n}}(\approx i),(\mathrm{b})$ one should be aware of it in conventional frp in systems with $D P_{\mathrm{n}}<100$, and (c) it is probably safe to ignore at higher $D P_{n}$. It has to be stressed here, however, that (although physically sensible!) these conclusions are only based on a limited amount of available data and that a possible additional mechanism of CLDP at higher chain lengths may complicate matters further. The situation for termination seems to be much clearer. Our recently proposed compositetermination model has independently been shown to present a good representation for the termination process in several different monomers. A generally applicable scaling law, reflecting the chain-length dependence of the individual rate coefficients, seems to apply to the dependence of $\left\langle k_{\mathrm{t}}\right\rangle$ on $D P_{\mathrm{n}}$ and is fairly insensitive to CLDP. For propagation, we have not yet succeeded in deriving a generally applicable scaling law for the variation of $\left\langle k_{\mathrm{p}}\right\rangle$ with $D P_{\mathrm{n}}$.

[1] M. Buback, M. Egorov, R.G. Gilbert, V. Kaminsky, O.F. Olaj, G.T. Russell, P. Vana, G. Zifferer, Macromol. Chem. Phys. 2002, 203, 2570.

[2] C. Barner-Kowollik, M. Buback, M. Egorov, T. Fukuda, A. Goto, O.F. Olaj, G.T. Russell, P. Vana, 
B. Yamada, P.B. Zetterlund, Prog. Polym. Sci. 2005, 30, 605.

[3] G.B. Smith, G.T. Russell, M. Yin, J.P.A. Heuts, Eur. Polym. J. 2005, 41, 225.

[4] G.B. Smith, J.P.A. Heuts, G.T. Russell, Macromol. Symp. 2005, 226, 133.

[5] J.P.A. Heuts, G.T. Russell, Eur. Polym. J. 2006, 42, 3.

[6] J.P.A. Heuts in Handbook of Radical Polymerization, K. Matyjaszewski, T.P. Davis, Eds., John Wiley \& Sons 2002, 1.

[7] G.B. Smith, G.T. Russell, J.P.A. Heuts, Macromol. Theory Simul. 2003, 12, 299.

[8] M. Buback, M. Egorov, T. Junkers, E. Panchencko, Macromol. Rapid Commun. 2004, 1004.

[9] M. Buback, E. Muller, G.T. Russell, J. Phys. Chem. A 2006, 110, 3222.

[10] R.X.E. Willemse, B.B.P. Staal, A.M. van Herk, S.C.J. Pierik, B. Klumperman, Macromolecules 2003, 36, 9797.

[11] O.F. Olaj, M. Zoder, P. Vana, A. Kornherr, I. Schnöll-Bitai, G. Zifferer, Macromolecules 2005, 38, 1944.

[12] H. Fischer, L. Radom, Angew. Chem. Int. Ed. 2001, 40, 1349.

[13] See, for example, S. Perrier, C. Barner-Kowollik, J.F. Quinn, P. Vana, T.P. Davis, Macromolecules 2002, 35, 8300.

[14] M.L. Coote, T.P. Davis, Prog. Polym. Sci. 1999, 24, 1217. 